\title{
Completely positive quantum stochastic convolution cocycles and their dilations
}

\author{
BY ADAM G. SKALSKI† \\ Department of Mathematics and Statistics, Lancaster University, Lancaster, LA1 4YF. \\ e-mail: a.skalski@lancaster.ac.uk
}

(Received 04 April 2006; revised 25 August 2006)

\begin{abstract}
Stochastic generators of completely positive and contractive quantum stochastic convolution cocycles on a $C^{*}$-hyperbialgebra are characterised. The characterisation is used to obtain dilations and stochastic forms of Stinespring decomposition for completely positive convolution cocycles on a $C^{*}$-bialgebra.
\end{abstract}

Stochastic (or Markovian) cocycles on operator algebras are basic objects of interest in quantum probability ([1]) and have been extensively investigated using quantum stochastic analysis (see [12]). There is also a well-developed theory of quantum Lévy processes, that is stationary, independent-increment, *-homomorphic processes on a *-bialgebra (see [6, 22] and references therein). Close examination of these two directions has naturally led to the notion of quantum stochastic convolution cocycle on a quantum group (or, more generally, on a coalgebra), as introduced and investigated in [14] in an algebraic context, and in [17] in the analytic context of compact quantum groups. The main results have been summarised in [15]. Recent years have also seen an increased interest in the noncommutative generalisation of classical hypergroups ([3]), initiated by Chapovsky and Vainerman ([5]) and continued, for example, in the papers [10] and [11]. Compact quantum hypergroups differ from compact quantum groups in that their coproduct need not be multiplicative. However, it remains completely positive, which makes compact quantum hypergroups, or more generally $C^{*}$-hyperbialgebras, an appropriate category for the consideration of completely positive quantum stochastic convolution cocycles in a topological context (for the purely algebraic case see [7]). These cocycles may be viewed as natural counterparts of stationary, independent-increment processes on hypergroups. In [17] it is shown that, under certain regularity conditions, they satisfy coalgebraic quantum stochastic differential equations.

The aim of this paper is to prove dilation theorems for quantum stochastic convolution cocycles on a $C^{*}$-bialgebra. To this end it is first necessary to establish the detailed structure of the stochastic generators of completely positive and contractive convolution cocycles. We give a direct derivation of this exploiting ideas used in the analysis of standard quantum stochastic cocycles with finite-dimensional noise space ([13]). Once the structure of generators is known, one may consider question of dilating completely positive convolution

$\dagger$ Permanent address: Department of Mathematics, University of Łódź, ul. Banacha 22, 90-238 Łódź, Poland. 
cocycles to ${ }^{*}$-homomorphic ones. In the context of standard quantum stochastic cocycles this problem was treated in [8] and [9] (see also [2]). In the first of these papers it was shown that every Markov-regular completely positive and contractive cocycle arises as the image of a *homomorphic cocycle under a vacuum conditional expectation which averages out some dimensions of the quantum noise. In the second every Markov-regular completely positive and contractive cocycle was shown to be realisable as a composition of a *homomorphic cocycle with conjugation by a contraction operator process. This may be seen as a stochastic Stinespring decomposition. In this paper using the techniques of Goswami, Lindsay, Sinha and Wills we obtain analogous results for convolution cocycles on $C^{*}$-bialgebras. Multiplicativity of the coproduct is necessary here for obtaining dilations to ${ }^{*}$-homomorphic cocycles.

An alternative approach to the one presented in this paper would be to exploit more directly theorems known for standard quantum stochastic convolution cocycles and properties of the $R$-map introduced in [17]. In that paper the general form of the stochastic generators of completely positive and contractive convolution cocycles was determined by using a particular representation of the $C^{*}$-bialgebra in question and appealing directly to the results of [13], [18] and [20]; similar methods may be further used to obtain the dilation results presented here. One drawback of such an approach is that it involves using the deep Christensen-Evans theorem on quasi-innerness of derivations on represented $C^{*}$-algebras. Another is the necessity to reformulate the results of [8] and [9] in coordinate-free quantum stochastic calculus. This is also necessary for overcoming separability assumptions on the noise dimension spaces. Finally the von Neumann algebraic framework used in [8] would require nontrivial modifications. In sum, the method presented here has the advantage of being more elementary.

The paper is structured as follows. The first section contains the notation and elements of quantum stochastic analysis and operator space theory needed. In the second section $C^{*}$-hyperbialgebras are defined and the well-known technique of obtaining them from $C^{*}$ bialgebras via a noncommutative conditional expectation is recalled. Basic facts concerning quantum stochastic convolution cocycles and the structure of their stochastic generators in the completely positive and *-homomorphic cases are also included here. In the third section a more detailed description of the stochastic generators of Markov-regular, completely positive, contractive convolution cocycles, in terms of a certain tuple of objects, is derived. Dilations of such convolution cocycles on a $C^{*}$-bialgebra to *-homomorphic convolution cocycles are given in the fourth section, and the fifth section contains a stochastic Stinespring decomposition.

\section{Preliminaries}

In this section we introduce our notations and review results from quantum stochastic analysis relevant to the rest of the paper. We shall usually abbreviate quantum stochastic to $\mathrm{QS}$, completely positive to $\mathrm{CP}$ and completely positive, contractive to $\mathrm{CPC}$.

\section{Notation}

All vector spaces in this paper are complex and inner products are linear in their second argument. Algebraic tensor products are denoted by $\odot$.

Let $\mathrm{h}$ be a Hilbert space. Ampliations are denoted

$$
\iota_{\mathrm{h}}: B(\mathrm{H}) \longrightarrow B(\mathrm{H} \otimes \mathrm{h}), \quad T \longmapsto T \otimes I_{\mathrm{h}},
$$


and each vector $\xi \in \mathrm{h}$ defines operators

$$
E_{\xi}: \mathrm{H} \longrightarrow \mathrm{H} \otimes \mathrm{h}, \quad v \longmapsto v \otimes \xi \text { and } E^{\xi}=\left(E_{\xi}\right)^{*},
$$

generalising Dirac's bra-ket notation:

$$
E_{\xi}=I_{\mathrm{H}} \otimes|\xi\rangle \text { and } E^{\xi}=I_{\mathrm{H}} \otimes\langle\xi| .
$$

The particular Hilbert space $\mathrm{H}$ will always be clear from the context. For a subspace $E$ of h, $\mathcal{O}(E)$ will denote the vector space of linear operators in $\mathrm{h}$ with domain $E$.

Finally, for a function $f: \mathbb{R}_{+} \rightarrow \mathrm{h}$ and subinterval $I$ of $\mathbb{R}_{+}, f_{I}$ denotes the function $\mathbb{R}_{+} \rightarrow \mathrm{h}$ which agrees with $f$ on $I$ and is zero outside $I$ (cf. standard indicator-function notation). This convention also applies to vectors, by viewing them as constant functionsfor example

$$
\xi_{[s, t]}, \text { for } \xi \in \mathrm{h} \text { and } 0 \leqslant s<t .
$$

\section{Matrix spaces}

For an introduction to the theory of operator spaces we refer to [4]. For this paper it is sufficient to work with concrete operator spaces, that is closed subspaces of the space $B\left(\mathrm{~h} ; \mathrm{h}^{\prime}\right)$ of all bounded linear operators acting between Hilbert spaces $\mathrm{h}$ and $\mathrm{h}^{\prime}$. The spatial/minimal tensor product of operator spaces is denoted by $\otimes$, and when $\mathrm{V}, \mathrm{W}$ are operator spaces $C B(\mathrm{~V} ; \mathrm{W})$ denotes the space of all completely bounded maps from $\mathrm{V}$ to $\mathrm{W}$.

We need the concept of matrix spaces introduced by Lindsay and Wills in [20]. Let $\mathrm{V} \subset$ $B(\mathrm{~K})$ be an operator space and let $\mathrm{h}$ be a supplementary Hilbert space. The operator space:

$$
M_{\mathrm{h}}(\mathrm{V})=\left\{T \in B(\mathrm{~K} \otimes \mathrm{h}): E^{\xi^{\prime}} T E_{\xi} \in \mathrm{V} \text { for all } \xi, \xi^{\prime} \in \mathrm{h}\right\}
$$

is called the h-matrix space over $\mathrm{V}$. It is easy to see that $M_{\mathrm{h}}(\mathrm{V})$ contains the spatial tensor product $\mathrm{V} \otimes B(\mathrm{~h})$. Whenever $\mathrm{W} \subset B(\mathrm{H})$ is another operator space, and $\phi \in C B(\mathrm{~V} ; \mathrm{W})$, the map $\phi \otimes \operatorname{id}_{B(\mathrm{~h})}$ extends uniquely to a completely bounded map $\phi^{(\mathrm{h})}: M_{\mathrm{h}}(\mathrm{V}) \rightarrow M_{\mathrm{h}}(\mathrm{W})$ satisfying

$$
E^{\xi^{\prime}}\left(\phi^{(\mathrm{h})}(T)\right) E_{\xi}=\phi\left(E^{\xi^{\prime}} T E_{\xi}\right),
$$

for all $T \in M_{\mathrm{h}}(\mathrm{V}), \xi, \xi^{\prime} \in \mathrm{h}$. The map $\phi^{(\mathrm{h})}$ is called the $\mathrm{h}$-lifting of $\phi$.

\section{Fock space notations and $Q S$ processes}

Let $\mathrm{k}$ be a Hilbert space, called the noise dimension space. Then $\mathcal{F}_{\mathrm{k}}$ denotes the symmetric Fock space over $L^{2}\left(\mathbb{R}_{+} ; \mathrm{k}\right)$. Exponential vectors in $\mathcal{F}_{\mathrm{k}}$ are written $\varepsilon(f), f \in L^{2}\left(\mathbb{R}_{+} ; \mathrm{k}\right)$. The CCR flow of index $\mathrm{k}$, defined in terms of the second quantised shift on $L^{2}\left(\mathbb{R}_{+} ; \mathrm{k}\right)$, is denoted $\sigma=\left(\sigma_{t}\right)_{t \geqslant 0}$. Define

$$
\mathbb{S}_{\mathrm{k}}:=\operatorname{Lin}\left\{d_{[0, s[}: d \in \mathrm{k}, s \in \mathbb{R}_{+}\right\}
$$

and a corresponding subspace of $\mathcal{F}_{\mathrm{k}}$ :

$$
\mathcal{E}_{\mathrm{k}}:=\operatorname{Lin}\left\{\varepsilon(f): f \in \mathbb{S}_{\mathrm{k}}\right\} .
$$

When the space $\mathrm{k}$ is clear from the context we will simply write $\mathcal{F}, \mathbb{S}$ and $\mathcal{E}$. Elements of $\mathcal{E}$ will play the role of test functions. For a subspace $E$ of $\mathrm{k}$ the following notation will be employed:

$$
\widehat{E}:=\operatorname{Lin}\{\widehat{\mathrm{c}}: \mathrm{c} \in \mathrm{E}\}, \text { where } \widehat{\mathrm{c}}:=\left(\begin{array}{l}
1 \\
\mathrm{c}
\end{array}\right) \in \widehat{\mathrm{h}}:=\mathbb{C} \oplus \mathrm{h}
$$


Two further useful notations are

$$
e_{0}=\left(\begin{array}{l}
1 \\
0
\end{array}\right) \in \widehat{\mathrm{k}} \text { and } \Delta^{Q S}=\left[\begin{array}{ll}
0 & \\
& I_{\mathrm{k}}
\end{array}\right] \in B(\widehat{\mathrm{k}}) .
$$

Let $\mathrm{h}$ be an additional Hilbert space. By an $\mathrm{h}$-operator process we understand a family $X=\left(X_{t}\right)_{t \geqslant 0}$ of operators on $\mathrm{h} \otimes \mathcal{F}$, each having the (dense) domain $\mathrm{h} \odot \mathcal{E}$, being weakoperator measurable in $t$ and adapted to the natural Fock-space operator filtration. Thus $X: \mathbb{R}_{+} \rightarrow \mathcal{O}(\mathrm{h} \odot \mathcal{E}), t \mapsto X_{t} \xi$ is weakly measurable for all $\xi \in \mathrm{h} \odot \mathcal{E}$ and, for each $t \geqslant 0$, $\zeta \in \mathrm{h}, X_{t}(\zeta \otimes \varepsilon(f))=X(t)\left(\zeta \otimes \varepsilon\left(\left.f\right|_{[0, t[}\right)\right) \otimes \varepsilon\left(\left.f\right|_{[t, \infty[}\right)$ for some operator $X(t) \in \mathcal{O}\left(\mathrm{h} \odot \mathcal{E}_{[0, t[}\right)$, where $\mathcal{E}_{[0, t[}$ is defined as $\mathcal{E}$ is, except that $\mathbb{R}_{+}$is replaced by $[0, t$. The linear space of all $\mathrm{h}$ operator processes is denoted $\mathbb{P}(\mathrm{h} \odot \mathcal{E})$, or $\mathbb{P}(\mathcal{E})$ if $\mathrm{h}=\mathbb{C}$, with subscripts on the $\mathcal{E}$ when necessary for avoiding ambiguity. A process $X \in \mathbb{P}(\mathrm{h} \odot \mathcal{E})$ is called weakly regular if for each $\xi, \xi^{\prime} \in \mathrm{h} \odot \mathcal{E}$, the scalar-valued function

$$
t \longmapsto\left\langle\xi^{\prime}, X_{t} \xi\right\rangle, \quad t \in \mathbb{R}_{+},
$$

is locally bounded. It is called bounded if $X_{t}$ is a bounded operator for each $t \geqslant 0$ (in such a case $X_{t}$ is usually identified with its continuous extension to the whole of $\mathrm{h} \otimes \mathcal{F}$ ).

Now let $\mathrm{V}$ and $\mathrm{W}$ be operator spaces with $\mathrm{W} \subset B(\mathrm{~h})$. A linear map $k$ from $\mathrm{V}$ to $\mathbb{P}(\mathrm{h} \odot \mathcal{E})$ is called a process on $\mathrm{V}$ with values in $\mathrm{W}$ if, for each $v \in \mathrm{V}, k(v)$ is an h-operator process and, for each $f, g \in \mathbb{S}, t \geqslant 0$ and $v \in \mathrm{V}$, the operator $E^{\varepsilon(f)} k_{t}(v) E_{\varepsilon(g)}$ belongs to W. Here W will usually be either $\mathrm{V}$ or $\mathbb{C}$.

The vector space of all processes on $\mathrm{V}$ with values in $\mathrm{W}$ is written $\mathbb{P}(\mathrm{V} ; \mathrm{W}, \mathcal{E})$ (this corresponds to the notation $\mathbb{P}(\mathrm{V} \rightarrow \mathrm{W}: \mathrm{h} \odot \mathcal{E})$ used in [17]); when $\mathrm{W}=\mathbb{C}$ we simply write $\mathbb{P}(\mathrm{V} ; \mathcal{E})$. We say that $k \in \mathbb{P}(\mathrm{V} ; \mathrm{W}, \mathcal{E})$ is pointwise weakly regular if each $k(v)(v \in \mathrm{V})$ is weakly regular. It is completely bounded if, for each $v \in \mathrm{V}$, the process $k(v)$ is bounded and, for each $t \geqslant 0$, the map $k_{t}: \mathrm{V} \rightarrow B(\mathrm{~h} \otimes \mathcal{F})$ is completely bounded.

$Q S$ differential equations and standard QS cocycles

Let $\mathrm{V}, \mathrm{W}$ be operator spaces with $\mathrm{W} \subset B(\mathrm{~h})$ for some Hilbert space $\mathrm{h}$. For maps $\theta \in$ $C B(\mathrm{~V} ; \mathrm{W})$ and $\phi \in C B\left(\mathrm{~V} ; M_{\widehat{\mathrm{k}}}(\mathrm{V})\right)$ consider the quantum stochastic differential equation

$$
d k_{t}=\widehat{k}_{t} \circ \phi d \Lambda_{t}, \quad k_{0}=\iota_{\mathcal{F}} \circ \theta .
$$

By a weak solution of this equation we understand a process $k \in \mathbb{P}(\mathrm{V} ; \mathrm{W}, \mathcal{E})$ such that

$$
\left\langle\xi \otimes \varepsilon(f),\left(k_{t}(v)-\theta(v) 1_{\mathcal{F}}\right) \eta \otimes \varepsilon(g)\right\rangle=\int_{0}^{t}\left\langle\xi \otimes \varepsilon(f), k_{s}\left(E^{\widehat{f}(s)} \phi(v) E_{\hat{g}(s)}\right) \eta \otimes \varepsilon(g)\right| d s
$$

for all $t \geqslant 0, v \in \mathrm{V}, \xi, \eta \in \mathrm{h}$ and $f, g \in \mathbb{S}$. If there is a quantum stochastically integrable $\mathrm{h} \otimes \widehat{\mathrm{k}}$-process $K$ on $\mathrm{V}$ (see [12]), with domain $\mathrm{h} \otimes \widehat{\mathrm{k}} \odot \mathcal{E}$, satisfying

$$
E^{\zeta^{\prime}} K_{t}(v) E_{\zeta}=k_{t}\left(E^{\zeta^{\prime}} \phi(v) E_{\zeta}\right)
$$

for all $t \geqslant 0, \zeta, \zeta^{\prime} \in \widehat{\mathrm{k}}$ and $v \in \mathrm{V}$, then $k$ is called a strong solution. The equation $(1 \cdot 2)$ has a unique weakly regular weak solution, which is also a strong solution ([16], [18]). We denote it by $k^{\theta, \phi}$, or simply $k^{\phi}$ if $\mathrm{V}=\mathrm{W}$ and $\theta=\mathrm{id}$.

A completely bounded process $k \in \mathbb{P}(\mathrm{V} ; \mathrm{V}, \mathcal{E})$ is called a standard $Q S$ cocycle on $\mathrm{V}$ if, for $s, t \geqslant 0$,

$$
k_{t+s}=\hat{k}_{t} \circ \widetilde{\sigma}_{t} \circ k_{s} \text { and } k_{0}=\iota_{\mathcal{F}} \circ \mathrm{id} \mathrm{V}
$$

where $\hat{k}_{t}$ denotes an $\mathcal{F}_{[t, \infty[}$-lifting of $k_{t}$ and $\widetilde{\sigma}_{s}=\mathrm{id}_{\mathrm{h}} \bar{\otimes} \sigma_{s}$. It is said to be Markov-regular if 
its Markov semigroup $P: \mathbb{R}_{+} \rightarrow B(\mathrm{~V})$, defined by

$$
P_{t}(v)=E^{\varepsilon(0)} k_{t}(v) E_{\varepsilon(0)}, \quad t \geqslant 0, v \in \mathrm{V},
$$

is norm-continuous. Whenever $\phi \in C B\left(\mathrm{~V} ; M_{\widehat{\mathrm{k}}}(\mathrm{V})\right)$, the process $k^{\phi}$ is a Markov-regular weak standard QS cocycle (see $[\mathbf{1 2}, \mathbf{1 9}$; note however that what is here called a weak standard QS cocycle, there is simply called a quantum stochastic cocycle).

\section{2. $C^{*}$-hyperbialgebras and $Q S$ convolution cocycles}

In this section we describe the standard construction of $C^{*}$-hyperbialgebras via noncommutative conditional expectation, and give the definition and some properties of quantum stochastic convolution cocycles. We then relate quantum stochastic convolution cocycles on the respective $C^{*}$-hyperbialgebras.

\section{$C^{*}$-hyperbialgebras and the conditional expectation construction}

Definition 2.1. A unital $C^{*}$-algebra $A$ is called a $C^{*}$-hyperbialgebra if it is equipped with a unital completely positive map $\Delta: A \rightarrow A \otimes A$ (called a coproduct) and a character $\epsilon: A \rightarrow \mathbb{C}$ (called a counit) satisfying the following conditions:

$$
\begin{gathered}
\left(\Delta \otimes \mathrm{id}_{\mathrm{A}}\right) \circ \Delta=\left(\mathrm{id}_{\mathrm{A}} \otimes \Delta\right) \circ \Delta, \\
\left(\epsilon \otimes \mathrm{id}_{\mathrm{A}}\right) \circ \Delta=\left(\mathrm{id}_{\mathrm{A}} \otimes \epsilon\right) \circ \Delta=\mathrm{id}_{\mathrm{A}} .
\end{gathered}
$$

If additionally $\Delta$ is multiplicative then $\mathrm{A}$ is called a $C^{*}$-bialgebra (and may be thought of as a compact quantum semigroup with a neutral element).

The following construction, of new $C^{*}$-hyperbialgebras from old, was described explicitly (in the context of compact quantum hypergroups) in the papers [10] and [11], but its origins go back much further (see [5] and references therein). All known examples of noncommutative $C^{*}$-hyperbialgebras arise in this way from $C^{*}$-bialgebras.

Proposition 2.2. Let $(\mathrm{A}, \Delta, \epsilon)$ be a $C^{*}$-hyperbialgebra. Assume that $\widetilde{\mathrm{A}}$ is a unital $C^{*}$ subalgebra of $\mathrm{A}$ and that there exists a conditional expectation, that is a norm-one projection, $P$ from $\mathrm{A}$ onto $\widetilde{\mathrm{A}}$ satisfying the following identities:

$$
\left(P \otimes i d_{\mathrm{A}}\right) \circ \Delta \circ P=(P \otimes P) \circ \Delta=\left(i d_{\mathrm{A}} \otimes P\right) \circ \Delta .
$$

Then $(\widetilde{\mathrm{A}}, \widetilde{\Delta}, \widetilde{\epsilon})$ is a $C^{*}$-hyperbialgebra, where

$$
\widetilde{\Delta}=\left.(P \otimes P) \circ \Delta\right|_{\tilde{A}} \text { and } \widetilde{\epsilon}=\left.\epsilon\right|_{\tilde{A}} .
$$

Two particular cases of this construction are double coset bialgebras and Delsarte $C^{*}$ hyperbialgebras; they are described below.

Let $\left(\mathrm{A}_{1}, \Delta_{1}, \epsilon_{1}\right)$ and $\left(\mathrm{A}_{2}, \Delta_{2}, \epsilon_{2}\right)$ be $C^{*}$-bialgebras and assume that the latter is a quantum subsemigroup of the former. This means that there exists a unital *-homomorphism $\pi: A_{1} \rightarrow A_{2}$ which is surjective and intertwines the coalgebraic structures:

$$
(\pi \otimes \pi) \circ \Delta_{1}=\Delta_{2} \circ \pi, \quad \epsilon_{2} \circ \pi=\epsilon_{1} .
$$

Assume additionally that $A_{2}$ admits a Haar state; this means that there exists a state $\mu \in A_{2}^{*}$ such that for all $a \in \mathrm{A}_{2}$

$$
\left(\mu \otimes \operatorname{id}_{\mathrm{A}_{2}}\right)\left(\Delta_{2}(a)\right)=\mu(a) 1_{\mathrm{A}_{2}} .
$$


Define the following $C^{*}$-subalgebras of $\mathrm{A}_{1}$ :

$$
\begin{aligned}
\mathrm{A}_{1} / \mathrm{A}_{2}:= & \left\{a \in \mathrm{A}_{1}:\left(\operatorname{id}_{\mathrm{A}_{1}} \otimes \pi\right) \circ \Delta_{1}(a)=a \otimes 1\right\}, \\
\mathrm{A}_{2} \backslash \mathrm{A}_{1}:= & \left\{a \in \mathrm{A}_{1}:\left(\pi \otimes \mathrm{id}_{\mathrm{A}_{1}}\right) \circ \Delta_{1}(a)=1 \otimes a\right\}, \\
& \mathrm{A}_{2} \backslash \mathrm{A}_{1} / \mathrm{A}_{2}:=\mathrm{A}_{1} / \mathrm{A}_{2} \cap \mathrm{A}_{2} \backslash \mathrm{A}_{1},
\end{aligned}
$$

called respectively the algebras of right and left cosets of $\mathrm{A}_{2}$ and the double coset bialgebra.

It can be checked that the map $P: A_{1} \rightarrow \widetilde{A}:=A_{2} \backslash A_{1} / A_{2}$ defined by

$$
P(a)=\left((\mu \circ \pi) \otimes \operatorname{id}_{\mathrm{A}_{1}} \otimes(\mu \circ \pi)\right)\left(\Delta_{1} \otimes \operatorname{id}_{\mathrm{A}_{1}}\right) \Delta_{1}(a), \quad a \in \mathrm{A}_{1},
$$

satisfies the conditions given in Proposition 2.2. Its action may be understood as averaging (twice) over the quantum subsemigroup; this construction is common in the theory of classical hypergroups ([3]).

Let now $(\mathrm{A}, \Delta, \epsilon)$ be a $C^{*}$-bialgebra and assume that a compact group $\Gamma$ acts (continuously with respect to the topology of pointwise convergence) on $\mathrm{A}$ by $C^{*}$-algebra automorphisms satisfying

$$
(\gamma \otimes \gamma) \circ \Delta=\Delta \circ \gamma, \quad \epsilon \circ \gamma=\epsilon, \quad \gamma \in \Gamma .
$$

Let $\widetilde{\mathrm{A}}$ be the fixed point subalgebra, $\widetilde{\mathrm{A}}:=\left\{a \in \mathrm{A}:{ }_{\gamma \in \Gamma} \gamma(a)=a\right\}$. It is easily checked that the map $P: \mathrm{A} \rightarrow \widetilde{\mathrm{A}}$ given by

$$
P(a)=\int_{\Gamma} \gamma(a) d \gamma, \quad a \in \mathrm{A},
$$

where $d \gamma$ denotes the normalised Haar measure on $\Gamma$, satisfies the assumptions of Proposition 2.2. The resulting $C^{*}$-hyperbialgebra is called a Delsarte $C^{*}$-hyperbialgebra.

Given a $C^{*}$-hyperbialgebra $\mathrm{A}$, each operator space $\mathrm{V}$ determines a map

$$
R_{\mathrm{V}}: C B(\mathrm{~A} ; \mathrm{V}) \longrightarrow C B(\mathrm{~A} ; \mathrm{A} \otimes \mathrm{V}), \quad \varphi \longmapsto\left(\mathrm{id}_{\mathrm{A}} \otimes \varphi\right) \circ \Delta .
$$

When $\mathrm{V}=\mathbb{C}$ we write $R$ instead of $R_{\mathbb{C}}$.

QS convolution cocycles

The following definition originates in [14] and is inspired by the theory of quantum Lévy processes. Let $\mathrm{A}$ be a $C^{*}$-hyperbialgebra.

Definition 2.3. A $Q S$ convolution cocycle on $\mathrm{A}$, with noise dimension space $\mathrm{k}$, is a completely bounded process $l \in \mathbb{P}(\mathrm{A} ; \mathcal{E})$ such that, for $s, t \geqslant 0$,

$$
l_{s+t}=\left(l_{s} \otimes\left(\sigma_{s} \circ l_{t}\right)\right) \circ \Delta \text { and } l_{0}=\iota_{\mathcal{F}} \circ \epsilon .
$$

The first of these conditions is referred to as the convolution increment property.

A QS convolution cocycle $l$ is said to be Markov-regular if its Markov convolution semigroup of functionals $\lambda: \mathbb{R}_{+} \rightarrow \mathrm{A}^{*}$, defined by

$$
\lambda_{t}(a)=\left\langle\varepsilon(0), l_{t}(a) \varepsilon(0)\right\rangle, \quad t \geqslant 0, a \in \mathrm{A},
$$

is norm-continuous.

For $\varphi \in C B(\mathrm{~A} ; B(\widehat{\mathrm{k}}))$ we consider coalgebraic QS differential equations of the form

$$
d l_{t}=l_{t} \star d \Lambda_{\varphi}(t)=\left(l_{t} \star_{\pi} \varphi\right) d \Lambda_{t}, \quad l_{0}=\iota_{\mathcal{F}} \circ \epsilon,
$$

where $\pi$ indicates a tensor flip exchanging the order of $\widehat{k}$ and $\mathcal{F}$. In fact the above equation may also be written as an equation of the type (1.2), with $\phi=R_{B(\widehat{\mathrm{k}})} \varphi$ and $\theta=\epsilon$. The unique 
solution of (2.2) will be denoted by $l^{\varphi}$. The process $l^{\varphi} \in \mathbb{P}(\mathrm{A} ; \mathcal{E})$ is a Markov-regular weak QS convolution cocycle. For full discussion of the precise meaning of (2.2), weak QS convolution cocycles and relations between the equation (2.2) and equations of the type (1.2) we refer to [16] and [17]. The next two propositions are proved in [17] by applying the semigroup decompositions of cocycles and convolution cocycles.

Proposition 2.4. Let $l=l^{\varphi}$ and $k=k^{\phi}$ where $\varphi \in C B(\mathrm{~A} ; B(\widehat{\mathrm{k}}))$ and $\phi=R_{B(\widehat{\mathrm{k}})} \varphi$. Then the process $l$ is completely bounded (respectively, completely positive and contractive) if and only if $k$ is, and if $l$ and $k$ are completely bounded then

$$
k_{t}=R_{B(\mathcal{F})} l_{t}, \quad t \in \mathbb{R}_{+} .
$$

PROPOSITION 2.5. Let $k=R_{B(\mathcal{F})}$ l where $l$ is a completely bounded process in $\mathbb{P}(\mathrm{A} ; \mathcal{E})$. Then $l$ is a $Q S$ convolution cocycle if and only if $k$ is a standard QS cocycle on A, and in this case $l$ is Markov-regular if and only if $k$ is.

Application of these results to the characterisation of the generators of Markov-regular CPC QS cocycles, and *-homomorphic QS cocycles, given in [20] and [21] respectively, leads to the following results.

THEOREM $2.6([17])$. Let $\mathrm{A}$ be a $C^{*}$-hyperbialgebra and $l \in \mathbb{P}\left(\mathrm{A} ; \mathcal{E}_{\mathrm{k}}\right)$. Then the following are equivalent:

(i) $l$ is a Markov-regular, completely positive and contractive $Q S$ convolution cocycle;

(ii) $l=l^{\varphi}$ where $\varphi \in C B(\mathrm{~A} ; B(\widehat{\mathrm{k}}))$ satisfies $\varphi(1) \leqslant 0$ and may be decomposed as follows:

$$
\varphi(a)=\psi(a)-\epsilon(a)\left(\Delta^{Q S}+\left|e_{0}\right\rangle\langle\chi|+| \chi\rangle\left\langle e_{0}\right|\right), \quad a \in \mathrm{A},
$$

for some completely positive map $\psi: A \rightarrow B(\widehat{\mathrm{k}})$ and vector $\chi \in \widehat{\mathrm{k}}$.

THEOREM 2.7 ([17]). Let $\mathrm{A}$ be a $C^{*}$-bialgebra and let $l=l^{\varphi}$ where $\varphi \in C B(\mathrm{~A} ; B(\widehat{\mathrm{k}}))$. Then the following are equivalent:

(i) $l$ is *-homomorphic;

(ii) $\varphi$ satisfies

$$
\varphi\left(a^{*} b\right)=\epsilon(a)^{*} \varphi(b)+\epsilon(b) \varphi(a)^{*}+\varphi(a)^{*} \Delta^{Q S} \varphi(b), \quad a, b \in \mathrm{A} .
$$

QS convolution cocycles and the conditional expectation construction

We end this section by describing the connection between QS convolution cocycles on $C^{*}$-hyperbialgebras related by the construction given in Proposition $2 \cdot 2$.

Proposition 2.8. Let $(\widetilde{\mathrm{A}}, \widetilde{\Delta}, \widetilde{\epsilon})$ be the $C^{*}$-hyperbialgebra arising from a $C^{*}$ hyperbialgebra $(\mathrm{A}, \Delta, \epsilon)$ via the construction presented in Proposition $2 \cdot 2$, with associated conditional expectation $P$. Then there is a 1-1 correspondence between $Q S$ convolution cocycles on $\widetilde{\mathrm{A}}$ and $P$-invariant processes on $\mathrm{A}$ satisfying the convolution increment property and having initial condition given by the functional $\epsilon \circ P$.

Proof. Assume first that $\tilde{l} \in \mathbb{P}(\tilde{\mathrm{A}} ; \mathcal{E})$ is a QS convolution cocycle and define $l \in \mathbb{P}(\mathrm{A} ; \mathcal{E})$ by

$$
l_{t}=\tilde{l}_{t} \circ P, \quad t \geqslant 0
$$

Then clearly $l_{0}(a)=\epsilon(P(a))$ for all $a \in \mathrm{A}$, and $l$ is $P$-invariant. It remains to check it is a 
convolution increment process. Choose $t, s \geqslant 0$ and compute:

$$
\begin{aligned}
l_{s+t}=\widetilde{l}_{s+t} \circ P & =\left(\widetilde{l}_{s} \otimes\left(\sigma_{s} \circ \widetilde{l}_{t}\right)\right) \circ \widetilde{\Delta} \circ P \\
& =\left(\widetilde{l}_{s} \otimes\left(\sigma_{s} \circ \widetilde{l}_{t}\right)\right) \circ(P \otimes P) \circ \Delta P \\
& =\left(\widetilde{l_{s}} \otimes\left(\sigma_{s} \circ \widetilde{l}_{t}\right)\right) \circ(P \otimes P) \circ \Delta=\left(l_{s} \otimes\left(\sigma_{s} \circ l_{t}\right)\right) \circ \Delta .
\end{aligned}
$$

Conversely, let $l \in \mathbb{P}(\mathrm{A} ; \mathcal{E})$ be a $P$-invariant convolution increment process, with initial condition given by $\epsilon \circ P$. Then the process $\widetilde{l} \in \mathbb{P}(\widetilde{A} ; \mathcal{E})$, defined simply by the restriction of $l$, is a $\mathrm{QS}$ convolution cocycle on $\widetilde{\mathrm{A}}$ - again the only thing to be checked is the convolution increment property: for all $s, t \geqslant 0, a \in \widetilde{\mathrm{A}}$,

$$
\begin{aligned}
\widetilde{l}_{s+t}(a) & =l_{s+t}(a) \\
& =\left(l_{s} \otimes\left(\sigma_{s} \circ l_{t}\right)\right)(\Delta(a)) \\
& =\left(l_{s} \otimes\left(\sigma_{s} \circ l_{t}\right)\right)(\Delta(P a)) \\
& =\left(l_{s} \otimes\left(\sigma_{s} \circ l_{t}\right)\right)((P \otimes P) \circ \Delta \circ P)(a)=\left(\widetilde{l}_{s} \otimes\left(\sigma_{s} \circ \widetilde{l}_{t}\right)\right)(\widetilde{\Delta}(a)) .
\end{aligned}
$$

Markov-regularity is clearly preserved in the above correspondence.

If $\epsilon=\epsilon \circ P$ (as is the case for Delsarte $C^{*}$-hyperbialgebras, but usually not for double coset bialgebras), then the processes $l$ arising in the proof of the above theorem are obviously QS convolution cocycles. Assuming this is the case, it is easily checked that if $\varphi \in C B(\widetilde{\mathrm{A}} ; B(\widehat{\mathrm{k}}))$ then $\widetilde{l}:=l^{\varphi} \in \mathbb{P}(\widetilde{\mathrm{A}} ; \mathcal{E})$ corresponds to the process $l^{\varphi \circ P} \in \mathbb{P}(\mathrm{A} ; \mathcal{E})$. There is an analogous correspondence on the level of weak QS convolution cocycles.

In [17] a variety of examples of $C^{*}$-bialgebras is presented, and *-homomorphic QS convolution cocycles on them are given alternative interpretations.

\section{Generator of CPC QS convolution cocycle}

In this section we consider the detailed structure of the stochastic generators of CPC QS convolution cocycles. Our approach is direct, following ideas used in the study of CPC standard QS cocycles. The crucial analysis was carried out in [13], with extension to infinite dimensional noise done in [18] and [20] (see also [2]).

Adapting arguments used in [13] requires some care, and the $R$-map introduced in (2.1) is an indispensable tool. A straightforward approach to complete positivity for QS convolution cocycles leads to nontrivial considerations of the proper convolution-counterpart of conditional complete positivity, and here the $R$-map does not appear to be helpful. However, nonnegative-definite kernels taking values in a $C^{*}$-algebra do behave well under the $R$-map, as will be seen in the proof of the next proposition.

For the rest of this section $\mathrm{A}$ denotes a fixed $C^{*}$-hyperbialgebra. For any $\tau \in B(\mathrm{~A})$ define $\partial \tau: \mathrm{A} \times \mathrm{A} \rightarrow \mathrm{A}$ by

$$
\partial \tau\left(a_{1}, a_{2}\right)=\tau\left(a_{1}^{*} a_{2}\right)-a_{1}^{*} \tau\left(a_{2}\right)-\tau\left(a_{1}^{*}\right) a_{2}+a_{1}^{*} \tau(1) a_{2}, \quad a_{1}, a_{2} \in \mathrm{A} .
$$

By analogy, for any $f \in \mathrm{A}^{*}$ define $\partial_{\epsilon} f: \mathrm{A} \times \mathrm{A} \rightarrow \mathbb{C}$ by

$$
\partial_{\epsilon} f\left(a_{1}, a_{2}\right)=f\left(a_{1}^{*} a_{2}\right)-\epsilon\left(a_{1}^{*}\right) f\left(a_{2}\right)-f\left(a_{1}^{*}\right) \epsilon\left(a_{2}\right)+\epsilon\left(a_{1}^{*}\right) f(1) \epsilon\left(a_{2}\right), \quad a_{1}, a_{2} \in \mathrm{A} .
$$

If $(\rho, \mathbf{K})$ is a representation of $\mathbf{A}$, a map $\delta: \mathbf{A} \rightarrow B(\mathbb{C} ; \mathbf{K})$ is called a $(\rho, \epsilon)$-derivation if for all $a_{1}, a_{2} \in \mathrm{A}$

$$
\delta\left(a_{1} a_{2}\right)=\rho\left(a_{1}\right) \delta\left(a_{2}\right)+\delta\left(a_{1}\right) \epsilon\left(a_{2}\right) .
$$


Observe that if $\varphi \in C B\left(\mathrm{~A} ; B(\widehat{\mathrm{k}})\right.$ and $l^{\varphi}$ is completely positive then it is easily verified that it is contractive too if and only if

$$
\varphi(1) \leqslant 0
$$

We need to start with the finite-dimensional situation. The key fact is the following result, corresponding to [13, theorem $4 \cdot 1]$.

Lemma 3.1. Assume that $\mathrm{k}$ is finite dimensional. Let $\varphi \in C B(\mathrm{~A} ; B(\widehat{\mathrm{k}}))$ and suppose that the (weak) QS convolution cocycle $l:=l^{\varphi} \in \mathbb{P}\left(\mathrm{A} ; \mathcal{E}_{\mathrm{k}}\right)$ is $C P C$. Then there exist a unital $*_{\text {-representation }}(\rho, \mathrm{K})$ of $\mathrm{A}$, a $(\rho, \epsilon)$-derivation $\delta: \mathrm{A} \rightarrow B(\mathbb{C} ; \mathrm{K})$, an operator $D \in B(\mathrm{k} ; \mathrm{K})$ and a vector $d \in \mathrm{k}$ such that

$$
\varphi(a)=\left[\begin{array}{cc}
\lambda(a) & \epsilon(a)\langle d|+\delta^{\dagger}(a) D \\
\epsilon(a)|d\rangle+D^{*} \delta(a) & D^{*} \rho(a) D-\epsilon(a) I_{\mathrm{k}}
\end{array}\right], \quad a \in \mathrm{A},
$$

where the functional $\lambda$ is real,

$$
\partial_{\epsilon} \lambda\left(a_{1}, a_{2}\right)=\delta\left(a_{1}\right)^{*} \delta\left(a_{2}\right), \quad a_{1}, a_{2} \in \mathrm{A},
$$

and the following minimality condition holds:

$$
\mathrm{K}=\overline{\operatorname{Lin}}\{\delta(a) 1+\rho(a) D c: a \in \mathrm{A}, c \in \mathrm{k}\} .
$$

If $\left(\mathrm{K}^{\prime}, \rho^{\prime}, \delta^{\prime}, D^{\prime}\right)$ is another quadruple satisfying the above conditions (except possibly the minimality condition), then there exists a unique isometry $V: \mathrm{K} \rightarrow \mathrm{K}^{\prime}$ such that

$$
\delta^{\prime}(a)=V \delta(a), \quad \rho^{\prime}(a) V=V \rho(a), \quad D^{\prime}=V D, \quad a \in \mathrm{A} .
$$

Proof. The proof is a modification of the argument used in the proof of lemma 4.5 in [13], where $\mathrm{k}$ is taken to be $\mathbb{C}^{d}$. Write $\varphi$ in block matrix form:

$$
\left[\begin{array}{cc}
\lambda & \tilde{\eta} \\
\eta & \sigma-\epsilon(\cdot) I_{\mathrm{k}}
\end{array}\right] \text {. }
$$

By Propositions 2.4 and $2 \cdot 5, k=R_{B\left(\mathcal{F}_{\mathrm{k}}\right)} l$ is a CPC standard QS cocycle and $\phi=R_{B(\widehat{\mathrm{k}})} \varphi$ is real. Therefore $\varphi$ is real too, in particular $\tilde{\eta}=\eta^{\dagger}$, and $\phi$ has block matrix form

$$
\left[\begin{array}{cc}
\tau & \alpha^{\dagger} \\
\alpha & v-\iota
\end{array}\right]
$$

where $\tau=R \lambda, \alpha=R_{B(\mathbb{C} ; \mathrm{k})} \eta$ and $\nu=R_{B(\mathrm{k})} \sigma$. Now lemma 4.4 in [13] implies that the map $\Psi$ from $\mathrm{A} \times \mathrm{A}$ to $\mathrm{A} \otimes B(\widehat{\mathrm{k}})$, there identified with $M_{d+1}(\mathrm{~A})$, defined by

$$
\Psi\left(a_{1}, a_{2}\right)=\left[\begin{array}{cc}
\partial \tau\left(a_{1}, a_{2}\right) & \alpha^{\dagger}\left(a_{1}^{*} a_{2}\right)-a_{1}^{*} \alpha^{\dagger}\left(a_{2}\right) \\
\alpha\left(a_{1}^{*} a_{2}\right)-\alpha\left(a_{1}^{*}\right) a_{2} & v\left(a_{1}^{*} a_{2}\right)
\end{array}\right], \quad a_{1}, a_{2} \in \mathrm{A},
$$

is nonnegative-definite. Observe that if $\psi: \mathrm{A} \times \mathrm{A} \rightarrow B(\widehat{\mathrm{k}})$ is defined by the formula

$$
\psi\left(a_{1}, a_{2}\right)=\left[\begin{array}{cc}
\partial_{\epsilon} \lambda\left(a_{1}, a_{2}\right) & \eta^{\dagger}\left(a_{1}^{*} a_{2}\right)-\epsilon\left(a_{1}^{*}\right) \eta^{\dagger}\left(a_{2}\right) \\
\eta\left(a_{1}^{*} a_{2}\right)-\eta\left(a_{1}^{*}\right) \epsilon\left(a_{2}\right) & \sigma\left(a_{1}^{*} a_{2}\right)
\end{array}\right], \quad a_{1}, a_{2} \in \mathrm{A},
$$

then $\psi=\left(\epsilon \otimes \mathrm{id}_{B(\widehat{\mathrm{k}})}\right) \circ \Psi$. This in turn implies that $\psi$ is a nonnegative-definite kernel. Indeed, for any $n \in \mathbb{N}, a_{1}, \ldots, a_{n} \in \mathrm{A}$ and $T_{1}, \ldots, T_{n} \in B(\widehat{\mathrm{k}})$

$$
\sum_{i, j=1}^{n} T_{i}^{*} \psi\left(a_{i}, a_{j}\right) T_{j}=\left(\epsilon \otimes \operatorname{id}_{B(\widehat{\mathrm{k}})}\right)\left(\sum_{i, j=1}^{n}\left(1_{\mathrm{A}} \otimes T_{i}^{*}\right) \Psi\left(a_{i}, a_{j}\right)\left(1_{\mathrm{A}} \otimes T_{j}\right)\right) \geqslant 0,
$$

as $\left(1_{\mathrm{A}} \otimes T_{i}\right)^{*}=\left(1_{\mathrm{A}} \otimes T_{i}^{*}\right) \in \mathrm{A} \otimes B(\widehat{\mathrm{k}}), \epsilon$ is $\mathrm{CP}$, and $\Psi$ is nonnegative-definite. 
Now let $(K, \chi)$ be the minimal Kolmogorov pair associated with $\psi$. This means that $\chi$ is a map $A \rightarrow B(\widehat{k} ; \mathrm{K})$ satisfying

$$
\begin{aligned}
& \chi\left(a_{1}\right)^{*} \chi\left(a_{2}\right)=\psi\left(a_{1}, a_{2}\right), \quad a_{1}, a_{2} \in \mathrm{A}, \\
& \mathrm{K}=\overline{\operatorname{Lin}}\{\chi(a) \zeta: a \in \mathrm{A}, \zeta \in \widehat{\mathrm{k}}\} .
\end{aligned}
$$

Properties of $\psi$ imply that $\chi$ is linear and bounded. Write $\chi=[\delta \gamma]$, where $\delta \in B(\mathrm{~A} ; B(\mathbb{C} ; \mathrm{K}))$ and $\gamma \in B(\mathrm{~A} ; B(\mathrm{k} ; \mathrm{K}))$. Then, for any $a, b \in \mathrm{A}$,

$$
\delta(a)^{*} \delta(b)=\partial_{\epsilon} \lambda(a, b) \text { and } \gamma(a)^{*} \delta(b)=\eta\left(a^{*} b\right)-\eta\left(a^{*}\right) \epsilon(b) .
$$

Setting $a=b=1$ shows that $\delta(1)=0$. Now for $u \in$ A unitary, define

$$
\delta_{u}(a)=\delta(u a)-\delta(u) \epsilon(a), \quad \gamma_{u}(a)=\gamma(u a) \text { and } \chi_{u}=\left[\delta_{u} \gamma_{u}\right], \quad \text { for } a \in \mathrm{A} .
$$

A straightforward computation yields

$$
\chi_{u}\left(a_{1}\right)^{*} \chi_{u}\left(a_{2}\right)=\chi\left(a_{1}\right)^{*} \chi\left(a_{2}\right)
$$

The uniqueness of minimal Kolmogorov pairs implies the existence of a unique isometry $\rho(u): \mathrm{K} \rightarrow \mathrm{K}$ given by the formula

$$
\rho(u)(\delta(a) 1+\gamma(a) c)=\delta(u a) 1-\delta(u) \epsilon(a)+\gamma(u a) c, \quad a \in \mathrm{A}, c \in \mathrm{k} .
$$

It follows, by standard arguments, that

$$
\rho(a)(\delta(b) 1+\gamma(b) c)=\delta(a b) 1-\delta(a) \epsilon(b)+\gamma(a b) c, \quad a, b \in \mathrm{A}, c \in \mathrm{k},
$$

defines a bounded operator $\rho(a)$ on $\mathrm{K}$. Moreover, it is easily checked that the resulting map $\rho: A \rightarrow B(K)$ is indeed a *-representation of $A$. It immediately follows that $\delta$ is a $(\rho, \epsilon)$-derivation and also, from minimality and the identity $\delta(1)=0$, that $\rho$ is unital. Put $D=\gamma(1) \in B(\mathrm{k} ; \mathrm{K})$. Then $\gamma(a)=\rho(a) D$, and furthermore $\sigma(a)=D^{*} \rho(a) D$ and $\eta(a)=$ $\epsilon(a) \eta(1)+D^{*} \delta(a) 1$. This yields (3·2) with $d=\eta(1) 1$.

The second part of the lemma follows once more from uniqueness of the Kolmogorov construction.

The step from finite-dimensional to arbitrary noise dimension space follows in exactly the same way as for standard cocycles.

LEMMA 3.2. Assume that $\mathrm{k}$ is an arbitrary Hilbert space. Let $\varphi \in C B(\mathrm{~A} ; B(\widehat{\mathrm{k}}))$ and suppose that the (weak) QS convolution cocycle $l^{\varphi} \in \mathbb{P}\left(\mathrm{A} ; \mathcal{E}_{\mathrm{k}}\right)$ is $C P C$. Then the conclusions of Lemma 3.1 hold.

Proof. Let $\left\{\mathrm{k}_{\iota}: \iota \in \mathcal{I}\right\}$ be an indexing of the set of all finite-dimensional subspaces of $\mathrm{k}$, which is partially ordered by inclusion. As in [18] we consider finite-dimensional cut-offs of both $l^{\varphi}$ and $\varphi$ itself. For each $\iota \in \mathcal{I}$ denote by $\varphi_{\iota}$ the map $\mathrm{A} \rightarrow B\left(\widehat{\mathrm{k}_{\iota}}\right)$ given by the formula

$$
\varphi_{\iota}(a)=P_{\iota} \varphi(a) P_{\iota}, \quad a \in \mathrm{A},
$$

where $P_{\iota} \in B(\widehat{\mathrm{k}})$ is the orthogonal projection onto $\widehat{\mathrm{k}}_{\iota}$. Setting $l^{(\iota)}=l^{\varphi_{\iota}}, \mathcal{F}=\mathcal{F}_{\mathrm{k}_{\iota}}, \mathcal{E}^{\iota}=\mathcal{E}_{\mathrm{k}_{\iota}}$ and letting $\mathbb{E}_{l}$ denote the vacuum conditional expectation map from $B\left(\mathcal{F}_{\mathrm{k}}\right)$ to $B(\mathcal{F})$, it is easy to see that $l^{(\imath)} \in \mathbb{P}\left(\mathrm{A} ; \mathcal{E}^{l}\right)$ is a CPC QS convolution cocycle and that it satisfies

$$
l_{t}^{(\iota)}(a)=\mathbb{E}_{\iota}\left[l_{t}^{\varphi}(a)\right], \quad a \in \mathrm{A}, t \in \mathbb{R}_{+} .
$$


Lemma $3 \cdot 1$ yields quadruples $\left(\mathrm{K}_{\iota}, \rho_{\iota}, \delta_{l}, D_{\iota}\right)$, unique up to isometric isomorphism, such that for all $a \in \mathrm{A}$

$$
\varphi_{\iota}(a)=\left[\begin{array}{cc}
\lambda(a) & \epsilon(a)\left\langle d_{\iota}\right|+\delta_{\iota}^{\dagger}(a) D_{\iota} \\
\epsilon(a)\left|d_{\iota}\right\rangle+D_{\iota}^{*} \delta_{\iota}(a) & D_{\iota}^{*} \rho_{\iota}(a) D_{\iota}-\epsilon(a) I_{\iota}
\end{array}\right],
$$

where $I_{\iota}$ denotes the identity operator on $\mathrm{k}_{\iota}$.

Exploiting uniqueness one can construct an inductive limit $\mathrm{K}$ of the Hilbert spaces $\mathrm{K}_{\iota}$. Denote by $U_{\iota}$ the respective isometry $\mathrm{K}_{\iota} \rightarrow \mathrm{K}$. Then there is a unital *-representation $\rho$ of $\mathrm{A}$ on $\mathrm{K}$ and $\mathrm{a}(\rho, \epsilon)$-derivation $\delta: \mathrm{A} \rightarrow \boldsymbol{B}(\mathbb{C} ; \mathrm{K})$

$$
\rho(a) U_{\iota}=U_{\iota} \rho_{\iota}(a), \quad \delta(a)=U_{\iota} \delta_{\iota}(a)
$$

for all $\iota \in \mathcal{I}, a \in \mathrm{A}$. Similarly, for each $c \in \mathrm{k}$ there exists a vector $c_{D} \in \mathrm{K}$ such that

$$
c_{D}=U_{\iota} D_{\iota} c
$$

for all $\iota \in \mathcal{I}$ such that $c \in \mathrm{k}_{\iota}$. The map $c \mapsto c_{D}$ is linear; it remains to show that it is bounded. To this end observe that, for any $\iota \in \mathcal{I}$ such that $c \in \mathrm{k}_{\iota}$,

$$
\begin{aligned}
\left\langle\left(\begin{array}{l}
0 \\
c
\end{array}\right), \varphi(1)\left(\begin{array}{l}
0 \\
c
\end{array}\right)\right\rangle & =\left\langle\left(\begin{array}{l}
0 \\
c
\end{array}\right), \varphi_{\iota}(1)\left(\begin{array}{l}
0 \\
c
\end{array}\right)\right\rangle \\
& =\left\langle c,\left(D_{\iota}^{*} D_{\iota}-\epsilon(1) I_{\iota}\right) c\right\rangle=\left\|D_{\iota} c\right\|^{2}-\|c\|^{2}=\left\|c_{D}\right\|^{2}-\|c\|^{2},
\end{aligned}
$$

and inequality (3.1), being a consequence of the contractivity of $l^{\varphi}$, implies that $\left\|c_{D}\right\| \leqslant\|c\|$. The operator $D \in B(\mathrm{k} ; \mathrm{K})$ given by $D c=c_{D}$ completes the tuple whose existence we wished to establish. Minimality holds by construction.

Automatic innerness of $(\rho, \epsilon)$-derivations leads to the following theorem.

THEOREM 3.3. Let $\varphi \in C B(\mathrm{~A} ; B(\widehat{\mathrm{k}}))$, for a $C^{*}$-hyperbialgebra $\mathrm{A}$, and suppose that the weak $Q S$ convolution cocycle $l^{\varphi} \in \mathbb{P}\left(\mathrm{A} ; \mathcal{E}_{\mathrm{k}}\right)$ is completely positive and contractive. Then there exists a tuple $(\mathrm{K}, \rho, D, \xi, d, e, t)$ constisting of a unital *-representation $(\rho, \mathrm{K})$ of $\mathrm{A}$, a contraction $D \in B(\mathrm{k} ; \mathrm{K})$, vectors $\xi \in \mathrm{K}$ and $d, e \in \mathrm{k}$, and a real number $t$, such that

$$
\varphi(a)=\left[\begin{array}{cc}
\lambda(a) & \epsilon(a)\langle d|+\delta^{\dagger}(a) D \\
\epsilon(a)|d\rangle+D^{*} \delta(a) & D^{*} \rho(a) D-\epsilon(a) I_{\mathrm{k}}
\end{array}\right],
$$

$t=\lambda(1) \leqslant 0, d=\left(I_{\mathrm{k}}-D^{*} D\right)^{1 / 2} e,\|e\|^{2} \leqslant-t$ and, for all $a \in \mathrm{A}$,

$$
\delta(a)=(\rho(a)-\epsilon(a))|\xi\rangle, \quad \lambda(a)=\epsilon(a)\left(t-\|\xi\|^{2}\right)+\langle\xi, \rho(a) \xi\rangle .
$$

Proof. Lemma 3.2 gives the form (3.4) for some $\rho, \mathrm{K}, \delta$ and $D$. As all $(\rho, \epsilon)$-derivations are inner (see [17, appendix]), there exists $\xi \in \mathrm{K}$ such that

$$
\delta(a)=\rho(a)|\xi\rangle-\epsilon(a)|\xi\rangle .
$$

It remains to note that

$$
\varphi(1)=\left[\begin{array}{cc}
t & \langle d| \\
|d\rangle & D^{*} D-I_{\mathrm{k}}
\end{array}\right],
$$

and the condition $\varphi(1) \leqslant 0$ implies contractivity of $D$, negativity of $t$ and the existence of a vector $e \in \mathrm{k}$ satisfying all the conditions above (see the characterisation of positive 'block' matrices given in lemma $2 \cdot 1$ of [9]). 
Remarks. The converse is also true - if a map $\varphi \in C B(\mathrm{~A} ; B(\mathrm{k}))$ has the above form then $l^{\varphi}$ is CPC. This follows easily from Propositions 2.4 and 2.5 and theorem 2.4 of [20].

If $l^{\varphi}$ is also unital, then $\varphi(1)=0$ and (3.4) takes the form

$$
\varphi(a)=\left[\begin{array}{cc}
\lambda(a) & \delta^{\dagger}(a) D \\
D^{*} \delta(a) & D^{*} \rho(a) D-\epsilon(a) I_{\mathrm{k}}
\end{array}\right],
$$

with $D$ being an isometry. This corresponds exactly to the characterisation obtained in the purely algebraic case by Franz and Schürmann ([7]).

The characterisation in Theorem 3.3 yields, as announced in the beginning of this section, an alternative proof of the existence of the decomposition (2.4) of Theorem 2.6. Indeed, for $\varphi: \mathrm{A} \rightarrow B(\widehat{\mathrm{k}})$ of the form (3.4), define $S: \widehat{\mathrm{k}} \rightarrow \mathrm{K}$ by $S=[|\xi\rangle, D]$. Then

$$
\varphi(a)=S^{*} \rho(a) S+\left[\begin{array}{cc}
\lambda_{0}(a) & \epsilon(a)\left\langle d-D^{*} c\right| \\
\epsilon(a)\left|d-D^{*} c\right\rangle & -\epsilon(a) I_{\mathrm{k}}
\end{array}\right], \quad a \in \mathrm{A},
$$

where $\lambda_{0}(a)=\lambda(a)-\langle\xi, \rho(a) \xi\rangle$. Note that as $\partial_{\epsilon} \lambda_{0}\left(a_{1}, a_{2}\right)=0$ for any $a_{1}, a_{2} \in \mathrm{A}, \lambda_{0}=$ $\lambda_{0}(1) \epsilon$ - one can check that $\left(\lambda_{0}-\lambda_{0}(1) \epsilon\right)$ is an $(\epsilon, \epsilon)$-derivation and so is zero. The map $\psi: \mathrm{A} \rightarrow B(\widehat{\mathrm{k}})$ defined by

$$
\psi(a)=S^{*} \rho(a) S, \quad a \in \mathrm{A},
$$

is completely positive. Setting $\chi=\left(\begin{array}{l}\frac{1}{2} \lambda_{0}(1) \\ d-D^{*} c\end{array}\right)$ yields the required decomposition.

\section{Dilations to *-homomorphic QS convolution cocycles}

This section addresses the question of dilating a completely positive, contractive QS convolution cocycle on a $C^{*}$-bialgebra to a *-homomorphic QS convolution cocycle. It is closely patterned on [9]. From now on we assume that $\mathrm{A}$ is a $C^{*}$-bialgebra. Recall that this means that $\mathrm{A}$ is a $C^{*}$-hyperbialgebra whose coproduct is multiplicative. Let $\mathrm{k}_{0}$ be a closed subspace of a noise dimension space $\mathrm{k}$. The vacuum conditional expectation from $B\left(\mathcal{F}_{\mathrm{k}}\right)$ to $B\left(\mathcal{F}_{\mathrm{k}_{0}}\right)$ will be denoted by $\mathbb{E}_{0}$.

Definition 4.1. A (weak) QS convolution cocycle $j \in \mathbb{P}\left(\mathrm{A} ; \mathcal{E}_{\mathrm{k}}\right)$ is said to be a stochastic dilation of a (weak) QS convolution cocycle $l \in \mathbb{P}\left(\mathrm{A} ; \mathcal{E}_{\mathrm{k}_{0}}\right)$ if

$$
l_{t}=\mathbb{E}_{0} \circ j_{t}, \quad t \geqslant 0 .
$$

The following result follows in exactly the same way as its counterpart for standard cocycles $([\mathbf{9}$, lemma 1·2]).

Proposition 4.2. Let $\varphi \in C B(\mathrm{~A} ; B(\widehat{\mathrm{k}}))$ and $\psi \in C B\left(\mathrm{~A} ; B\left(\widehat{\mathrm{k}}_{0}\right)\right)$, and let $j=l^{\varphi} \in$ $\mathbb{P}\left(\mathrm{A} ; \mathcal{E}_{\mathrm{k}}\right)$ and $l=l^{\psi} \in \mathbb{P}\left(\mathrm{A} ; \mathcal{E}_{\mathrm{k}_{0}}\right)$ be the respective $Q S$ convolution cocycles. Then $j$ is $a$ stochastic dilation of $l$ if and only if $\psi(\cdot)=P_{0} \varphi(\cdot) P_{0}$, where $P_{0} \in B(\widehat{\mathrm{k}})$ denotes the orthogonal projection onto $\widehat{\mathrm{k}}_{0}$.

Generators of *-homomorphic cocycles may be described in the following way.

Proposition 4.3. Let $(\mathrm{K}, \rho, D, \xi, d, t)$ be a tuple as in Theorem 3.3 and let $\varphi$ be the map in $C B(\mathrm{~A} ; B(\widehat{\mathrm{k}}))$ given by the formulas (3.4) and (3.5). Then the (weak) QS convolution cocycle $l^{\varphi} \in \mathbb{P}\left(\mathrm{A} ; \mathcal{E}_{\mathrm{k}}\right)$ is *-homomorphic if and only if the following conditions hold:

(i) $D$ is a partial isometry;

(ii) $D d=0$;

(iii) $D D^{*} \in \rho(\mathrm{A})^{\prime}$; 
(iv) $t=-\|d\|^{2}$;

(v) $D D^{*} \delta=\delta$;

where $\delta$ is the $(\rho, \epsilon)$-derivation $a \mapsto\left(\rho(a)-\epsilon(a) I_{\mathrm{k}}\right)|\xi\rangle$.

Proof. In the language of Theorem 3.3, the structure relations (2.5) translate into the following identities:

$$
\begin{aligned}
& D^{*} \rho(a) D D^{*} \rho(b) D=D^{*} \rho(a b) D, \\
& D^{*} \delta(a b)+\epsilon(a b)|d\rangle=D^{*} \rho(a) D\left(D^{*} \delta(b)+\epsilon(b)|d\rangle\right)+D^{*} \delta(a) \epsilon(b)+\epsilon(a) \epsilon(b)|d\rangle, \\
& \lambda\left(a^{*} b\right)=\left\langle D^{*} \delta(a) 1+\epsilon(a) d, D^{*} \delta(b) 1+\epsilon(b) d\right\rangle+\lambda\left(a^{*}\right) \epsilon(b)+\epsilon\left(a^{*}\right) \lambda(b),
\end{aligned}
$$

for all $a, b \in \mathrm{A}$. As in proposition $3 \cdot 3$ of [9], this in turn may be shown to be equivalent to the conditions (i)-(v).

Remarks. Observe that the above characterisation excludes the possibility of obtaining exchange free dilations-it can be seen directly from (2.5) that if a Markov-regular *-homomorphic QS convolution cocycle is generated by a map having the form

$$
\left[\begin{array}{ll}
* & * \\
* & 0
\end{array}\right]
$$

then it is identically 0 . This uses the fact that $(\epsilon, \epsilon)$-derivations are trivial. As to creation/annihilation free dilations, they are possible only for those CPC QS convolution cocycles, whose generators have the form

$$
\left[\begin{array}{ll}
0 & 0 \\
0 & *
\end{array}\right]
$$

Moreover, $j$ is unital, as well as being *-homomorphic, if and only if (iii) and (v) are satisfied, $D$ is an isometry, $d=0$ and $t=0$.

As a consequence of Theorem 3.3 and Proposition 4.3, we obtain the existence of stochastic dilations.

THEOREM 4.4. Every Markov-regular CPC QS convolution cocycle on a $C^{*}$-bialgebra A admits a Markov-regular *-homomorphic stochastic dilation.

Proof. Let $l \in \mathbb{P}\left(\mathrm{A} ; \mathcal{E}_{\mathrm{k}_{0}}\right)$ be a Markov-regular CPC QS convolution cocycle. Then $l=$ $l^{\varphi}$ for some $\varphi \in C B\left(\mathrm{~A} ; B\left(\widehat{\mathrm{k}}_{0}\right)\right)$ and we can assume that $\varphi$ has matrix form (3.4) for a tuple (K, $\rho, D, \xi, d, e, t)$ with the properties described in Theorem 3.3. Let $\mathrm{k}_{1}, \mathrm{k}_{2}$ be Hilbert spaces, suppose that $d_{1} \in \mathrm{k}_{1}, d_{2} \in \mathrm{k}_{2}, D_{1} \in B\left(\mathrm{k}_{1} ; \mathrm{K}\right)$ (all as yet unspecified) and consider the map $\psi: \mathrm{A} \rightarrow B(\widehat{\mathrm{k}})$, where $\mathrm{k}:=\mathrm{k}_{0} \oplus \mathrm{k}_{1} \oplus \mathrm{k}_{2}$, given by

$$
\psi(a)=\left[\begin{array}{cccc}
\lambda(a) & \epsilon(a)\langle d|+\delta^{\dagger}(a) D & \epsilon(a)\left\langle d_{1}\right|+\delta^{\dagger}(a) D_{1} & \epsilon(a)\left\langle d_{2}\right| \\
\epsilon(a)|d\rangle+D^{*} \delta(a) & D^{*} \rho(a) D-\epsilon(a) I_{0} & D^{*} \rho(a) D_{1} & 0 \\
\epsilon(a)\left|d_{1}\right\rangle+D_{1}^{*} \delta(a) & D_{1}^{*} \rho(a) D & D_{1}^{*} \rho(a) D_{1}-\epsilon(a) I_{1} & 0 \\
\epsilon(a)\left|d_{2}\right\rangle & 0 & 0 & -\epsilon(a) I_{2}
\end{array}\right]
$$

( $a \in \mathrm{A}$ ), with $I_{i}$ denoting $I_{\mathrm{k}_{\mathrm{i}}}, i=0,1,2$. Now observe that $\psi$ can also be written in the form

$$
\psi(a)=\left[\begin{array}{cc}
\lambda(a) & \epsilon(a)\langle\widetilde{d}|+\delta^{\dagger}(a) \widetilde{D} \\
\epsilon(a)|\widetilde{d}\rangle+\widetilde{D}^{*} \delta(a) & \widetilde{D}^{*} \rho(a) \widetilde{D}-\epsilon(a) I_{\mathrm{k}}
\end{array}\right],
$$


where

$$
\widetilde{d}=\left(\begin{array}{l}
d \\
d_{1} \\
d_{2}
\end{array}\right) \in \mathrm{k} \text { and } \widetilde{D}=\left[\begin{array}{lll}
D & D_{1} & 0
\end{array}\right] \in B(\mathrm{k} ; \mathrm{K}) .
$$

As $\psi$ is clearly completely bounded, it generates a weak QS convolution cocycle $l^{\psi} \in$ $\mathbb{P}\left(\mathrm{A} ; \mathcal{E}_{\mathrm{k}}\right)$. It follows from Proposition $4 \cdot 2$ that $l^{\psi}$ is a stochastic dilation of $l^{\varphi} ;$ it remains to show that we can choose the parameters $\mathrm{k}_{1}, \mathrm{k}_{2}, d_{1}, d_{2}$ and $D_{1}$ so that $l^{\psi}$ is *-homomorphic.

To this end, it suffices to put $\mathrm{k}_{1}=\mathrm{K}, \mathrm{k}_{2}=\mathbb{C}$,

$$
D_{1}=\left(I_{1}-D D^{*}\right)^{\frac{1}{2}}, \quad d_{1}=-D e, \quad d_{2}=\sqrt{-\left(t+\|e\|^{2}\right)} .
$$

The above definitions make sense as $\|e\| \leqslant-t$ and $D$ is a contraction. It remains then to check properties (i)-(v) of Proposition 4.3. First note that

$$
\widetilde{D} \widetilde{D}^{*}=D D^{*}+I_{1}-D D^{*}=I_{1},
$$

which implies that conditions (i), (iii) and (v) are satisfied (one can easily check that $\widetilde{D}^{*} \widetilde{D}$ is a selfadjoint projection). Further we obtain (ii):

$$
\widetilde{D} \widetilde{d}=D\left(I_{0}-D^{*} D\right)^{\frac{1}{2}} e-\left(I_{1}-D D^{*}\right)^{\frac{1}{2}} D e=0 .
$$

Finally (iv) follows since

$$
\|\widetilde{d}\|^{2}=\left\|\left(I_{0}-D^{*} D\right)^{1 / 2} e\right\|^{2}+\|D e\|^{2}-\left(t+\|e\|^{2}\right)=-t .
$$

This completes the proof.

If $l$ is unital and the Hilbert space dimensions of $\mathrm{K}$ and $\overline{\operatorname{Ran}\left(\mathrm{I}_{\mathrm{K}}-\mathrm{DD}^{*}\right)}$ coincide, then $j$ may be chosen to be unital, with $\mathrm{k}_{1}=\mathrm{K}$ and $\mathrm{k}_{2}=\{0\}$ in the above.

\section{Stinespring theorem for QS convolution cocycles}

As the previous section was a variation on the theme of [9], this one addresses the convolution counterpart of the problem considered in [8] for standard QS cocycles. We shall show (in Theorem 5.3) that each Markov-regular, completely positive, contractive QS convolution cocycle has a Stinespring-like decomposition in terms of a *-homomorphic cocycle perturbed by a contractive process.

First we need some remarks on QS differential equations of the type:

$$
\mathrm{d} W_{t}=F_{t}\left(I_{\widehat{\mathrm{k}}} \otimes W_{t}\right) \mathrm{d} \Lambda_{t}, \quad W_{0}=I_{\mathcal{F}},
$$

where $F \in \mathbb{P}(\widehat{\mathrm{k}} \odot \mathcal{E})$ is a bounded process. We say that $W$ is a weak solution of the above equation if for all $f, g \in \mathbb{S}$ and $t \geqslant 0$

$$
\left\langle\varepsilon(f),\left(W_{t}-I_{\mathcal{F}}\right) \varepsilon(g)\right\rangle=\int_{0}^{t}\left\langle\hat{f}(s) \otimes \varepsilon(f), F_{s}\left(I_{\widehat{\mathrm{k}}} \otimes W_{s}\right)(\hat{g}(s) \otimes \varepsilon(g))\right\rangle d s .
$$

The solution of the above equation is given by the iteration procedure:

$$
\begin{aligned}
& X_{t}^{0}=I_{\mathcal{F}}, \quad X_{t}^{1}=\int_{0}^{t} F_{s}\left(I_{\widehat{\mathrm{k}}} \otimes X_{s}^{0}\right) d \Lambda_{s}, \ldots, X_{t}^{n+1}=\int_{0}^{t} F_{s}\left(I_{\widehat{\mathrm{k}}} \otimes X_{s}^{n}\right) d \Lambda_{s}, \ldots \\
& W_{t} \varepsilon(f):=\sum_{n=0}^{\infty} X_{t}^{n} \varepsilon(f) .
\end{aligned}
$$


Sufficient conditions for the above heuristics to be justified are that $F$ is strongly measurable and has locally uniform bounds; this is also sufficient for the uniqueness of strongly regular strong solutions of the equation ([8, proposition $3 \cdot 1])$. These conditions are clearly satisfied when

$$
F_{s}=\left(\operatorname{id}_{B(\widehat{\mathrm{k}})} \otimes l_{s}\right)(T), \quad s \geqslant 0,
$$

where $l$ is a Markov-regular, CPC QS convolution cocycle and $T \in B(\widehat{\mathrm{k}}) \otimes \mathrm{A}$.

Now let $j$ be a $*$-homomorphic QS convolution cocycle of the form $l^{\varphi}$, where $\varphi \in$ $C B(\mathrm{~A} ; B(\widehat{\mathrm{k}}))$, and let $T \in B(\widehat{\mathrm{k}}) \otimes \mathrm{A}$. Assume that $W \in \mathbb{P}(\mathcal{E})$ is a bounded solution to the equation

$$
\mathrm{d} W_{t}=\left(\mathrm{id}_{B(\widehat{\mathrm{k}})} \otimes j_{t}\right)(T)\left(I_{\widehat{\mathrm{k}}} \otimes W_{t}\right) \mathrm{d} \Lambda_{t}, \quad W_{0}=I_{\mathcal{F}} .
$$

We shall identify sufficient conditions for $W$ to be a contractive process later. The next question to be addressed is: when can we expect a process $k \in \mathbb{P}(\mathrm{A} ; \mathcal{E})$ defined by

$$
k_{t}(a)=j_{t}(a) W_{t}, \quad a \in \mathrm{A}, t \geqslant 0,
$$

to be a Markov-regular QS convolution cocycle?

The quantum Itô formula yields

$$
\begin{aligned}
\left\langle\varepsilon(f), k_{t}(a) \varepsilon(g)\right\rangle= & \left\langle j_{t}\left(a^{*}\right) \varepsilon(f), W_{t} \varepsilon(g)\right\rangle \\
= & \epsilon(a)\langle\varepsilon(f), \varepsilon(g)\rangle \\
& +\int_{0}^{t} \operatorname{ds}\left(\left\langle\tilde{j}_{s}\left(I_{\widehat{\mathrm{k}}} \otimes a^{*}\right)(\hat{f}(s) \otimes \varepsilon(f)), \tilde{j}_{s}(T) \widetilde{W}_{s}(\hat{g}(s) \otimes \varepsilon(g))\right\rangle\right. \\
& +\left\langle\tilde{j}_{s}\left(\phi\left(a^{*}\right)\right)(\hat{f}(s) \otimes \varepsilon(f)), \widetilde{W}_{s}(\hat{g}(s) \otimes \varepsilon(g))\right\rangle \\
& \left.+\left\langle\tilde{j}_{s}\left(\phi\left(a^{*}\right)\right)(\hat{f}(s) \otimes \varepsilon(f)),\left(\Delta^{Q S} \otimes I_{\mathcal{F}}\right) \tilde{j}_{s}(T) \widetilde{W}_{s}(\hat{g}(s) \otimes \varepsilon(g))\right\rangle\right)
\end{aligned}
$$

$(f, g \in \mathbb{S}, t \geqslant 0)$, where $\phi=R_{B(\widehat{\mathrm{k}})} \varphi, \tilde{j}_{s}=\left(\mathrm{id}_{B(\widehat{\mathrm{k}})} \otimes j_{s}\right)$ and $\tilde{W}_{s}=I_{\widehat{\mathrm{k}}} \otimes W_{s}$. Defining analogously $\tilde{k}_{s}=\left(\mathrm{id}_{B(\widehat{\mathrm{k}})} \otimes k_{s}\right)$ we see that the above equation may be written as

$$
\begin{aligned}
& \left\langle\varepsilon(f), k_{t}(a) \varepsilon(g)\right\rangle=\epsilon(a)\langle\varepsilon(f), \varepsilon(g)\rangle \\
& \quad+\int_{0}^{t} \mathrm{ds}\left(\left\langle\hat{f}(s) \otimes \varepsilon(f), \tilde{k}_{s}\left(\left(I_{\widehat{\mathrm{k}}} \otimes a\right) T+\phi(a)+\phi(a)\left(\Delta^{Q S} \otimes 1_{\mathrm{A}}\right) T\right)(\hat{g}(s) \otimes \varepsilon(g))\right\rangle\right) .
\end{aligned}
$$

The process $k$ is equal to $l^{\psi}$ for some $\psi \in C B(\mathrm{~A} ; B(\widehat{\mathrm{k}}))$ if and only if $\widetilde{\psi}:=\left(\psi \otimes \mathrm{id}_{\mathrm{A}}\right) \circ \Delta$ is given by

$$
a \longmapsto\left(I_{\widehat{\mathrm{k}}} \otimes a\right) T+\phi(a)+\phi(a)\left(\Delta^{Q S} \otimes 1_{\mathrm{A}}\right) T .
$$

Note that we need to work with the left version of the map $R$ introduced in $(2 \cdot 1)$ because of the tensor flip in the definition of the coalgebraic QS differential equation $(2 \cdot 2)$. Let $\tau=\left(\operatorname{id}_{B(\widehat{\mathrm{k}})} \otimes \epsilon\right)(T) \in B(\widehat{\mathrm{k}})$. Then $(5 \cdot 3)$ implies that

$$
\psi(a)=\epsilon(a) \tau+\varphi(a)\left(1+\Delta^{Q S} \tau\right)
$$

and so

$$
\widetilde{\psi}(a)=\tau \otimes a+\phi(a)+\phi(a)\left(\Delta^{Q S} \tau \otimes 1_{\mathrm{A}}\right) .
$$

Comparing (5.3) with $(5 \cdot 5)$ yields

$$
\left(I_{\widehat{\mathrm{k}}} \otimes a\right) T+\phi(a)\left(\Delta^{Q S} \otimes 1_{\mathrm{A}}\right) T=\tau \otimes a+\phi(a)\left(\Delta^{Q S} \tau \otimes 1_{\mathrm{A}}\right) .
$$


If $T=\tau \otimes 1_{\mathrm{A}}$ then this condition is automatically satisfied. If $j$ is unital, then $T=\tau \otimes 1_{\mathrm{A}}$ is also necessary for (5.6) to hold: put $a=1_{\mathrm{A}}$ and use $\phi\left(1_{\mathrm{A}}\right)=0$.

Observe that when $T=\tau \otimes 1_{\mathrm{A}}$ the equation (5.2) takes the simple form

$$
\mathrm{d} W_{t}=\left(\tau \otimes U_{t} W_{t}\right) \mathrm{d} \Lambda_{t}, \quad W_{0}=I_{\mathcal{F}},
$$

with $U_{t}=j_{t}(1)$. In this case the condition on $\tau$ assuring contractivity of $W$ is also particularly simple.

THEOREM 5.1. Let $j=l^{\varphi}$ where $\varphi \in C B(\mathrm{~A} ; B(\widehat{\mathrm{k}}))$ and $\mathrm{A}$ is a $C^{*}$-bialgebra. Suppose that $j$ is *-homomorphic and $\tau \in B(\widehat{\mathrm{k}})$ satisfies the condition

$$
\tau+\tau^{*}+\tau^{*} \Delta^{Q S} \tau \leqslant 0 .
$$

Then the equation (5.7), with $U_{t}:=j_{t}(1)$, has a unique contractive strong solution $W \in \mathbb{P}(\mathcal{E})$ (contractive means that each $W_{t}$ is a contraction). Moreover the process $W_{t}^{*} j_{t}(\cdot) W_{t}$ is equal to $l^{\theta}$, where

$$
\theta(a)=\epsilon(a)\left(\tau^{*}+\tau+\tau^{*} \Delta^{Q S} \tau\right)+\left(I_{\widehat{\mathrm{k}}}+\tau^{*} \Delta^{Q S}\right) \varphi(a)\left(I_{\widehat{\mathrm{k}}}+\Delta^{Q S} \tau\right), \quad a \in \mathrm{A} .
$$

Proof. The discussion before the theorem shows that the equation (5.7) has a unique strongly regular strong solution $W \in \mathbb{P}(\mathcal{E})$. The Itô formula yields, for $u=\sum_{i=1}^{k} \lambda_{i} \varepsilon\left(f_{i}\right)$, $k \in \mathbb{N}, \lambda_{1}, \ldots, \lambda_{k} \in \mathbb{C}, f_{1}, \ldots, f_{k} \in \mathbb{S}$

$$
\begin{aligned}
\left\langle W_{t} u, W_{t} u\right\rangle-\langle u, u\rangle= & \sum_{i, j=1}^{k} \overline{\lambda_{i}} \lambda_{j} \int_{0}^{t} \mathrm{ds}\left(\left\langle\hat{f}_{i}(s) \otimes W_{s} \varepsilon\left(f_{i}\right), \tau \hat{f}_{j}(s) \otimes U_{s} W_{s} \varepsilon\left(f_{j}\right)\right\rangle\right. \\
& +\left\langle\tau \hat{f}_{i}(s) \otimes U_{s} W_{s} \varepsilon\left(f_{i}\right), \hat{f}_{j}(s) \otimes W_{s} \varepsilon\left(f_{j}\right)\right\rangle \\
& \left.+\left\langle\tau \hat{f}_{i}(s) \otimes U_{s} W_{s} \varepsilon\left(f_{i}\right), \Delta^{Q s} \tau \hat{f}_{j}(s) \otimes U_{s} W_{s} \varepsilon\left(f_{j}\right)\right\rangle\right) .
\end{aligned}
$$

As $U_{s}=j_{s}(1)$ and $j$ is *-homomorphic, each $U_{s}$ is a projection. Therefore putting $x(s)=$ $\sum_{i=1}^{k} \lambda_{i} \hat{f}_{i}(s) \otimes U_{s} W_{s} \varepsilon\left(f_{i}\right), s \in[0, t]$, yields

$$
\left\langle W_{t} u, W_{t} u\right\rangle-\langle u, u\rangle=\int_{0}^{t} \mathrm{ds}\left\langle x(s),\left(\left(\tau+\tau^{*}+\tau^{*} \Delta^{Q S} \tau\right) \otimes I_{\mathcal{F}}\right) x(s)\right\rangle \leqslant 0 .
$$

It follows that $W$ is contractive.

The proof of the second part of the theorem is a combination of the considerations before its formulation and one more application of the Itô formula. Again let $f, g \in \mathbb{S}, t \geqslant 0, a \in \mathrm{A}$ and $T=\tau \otimes 1_{\mathrm{A}}$, let $\tilde{j}, \tilde{k}, \widetilde{W}$ and $\psi$ be defined as in the discussion before the theorem and set $\widetilde{\psi}=\left(\psi \otimes \mathrm{id}_{\mathrm{A}}\right) \circ \Delta$. Then

$$
\begin{aligned}
\left\langle\varepsilon(f), W_{t}^{*} j_{t}(a) W_{t} \varepsilon(g)\right\rangle= & \left\langle W_{t} \varepsilon(f), j_{t}(a) W_{t} \varepsilon(g)\right\rangle \\
= & \epsilon(a)\langle\varepsilon(f), \varepsilon(g)\rangle \\
& +\int_{0}^{t} \mathrm{ds}\left(\left\langle\widetilde{W}_{s}(\hat{f}(s) \otimes \varepsilon(f)), \tilde{k}_{s}(\widetilde{\psi}(a))(\hat{g}(s) \otimes \varepsilon(g))\right\rangle\right. \\
& +\left\langle\tilde{j}_{s}(T) \widetilde{W}_{s}(\hat{f}(s) \otimes \varepsilon(f)), \tilde{j}_{s}\left(I_{\widehat{\mathrm{k}}} \otimes a\right) \widetilde{W}_{s}(\hat{g}(s) \otimes \varepsilon(g))\right\rangle \\
& \left.+\left\langle\tilde{j}_{s}(T) \widetilde{W}_{s}(\hat{f}(s) \otimes \varepsilon(f)),\left(\Delta^{Q S} \otimes I_{\mathcal{F}}\right) \tilde{k}_{s}(\widetilde{\psi}(a))(\hat{g}(s) \otimes \varepsilon(g))\right\rangle\right) .
\end{aligned}
$$

Finally, (5.4) yields

$$
\begin{aligned}
\left\langle\varepsilon(f), W_{t}^{*} j_{t}(a) W_{t} \varepsilon(g)\right\rangle= & \epsilon(a)\langle\varepsilon(f), \varepsilon(g)\rangle \\
& +\int_{0}^{t} \mathrm{ds}\left\langle\hat{f}(s) \otimes \varepsilon(f), \widetilde{W}_{s}^{*} \tilde{j}_{s}(\widetilde{\theta}(a)) \widetilde{W}_{s}(\hat{g}(s) \otimes \varepsilon(g))\right\rangle .
\end{aligned}
$$

where $\widetilde{\theta}=\left(\theta \otimes \mathrm{id}_{\mathrm{A}}\right) \circ \Delta$. This completes the proof. 
For each $t \geqslant 0$ denote the orthogonal projection from $\mathcal{F}$ onto $\mathcal{F}_{[t, \infty[}$ by $P_{\mathrm{k},[t, \infty[}$. The following result may be proved by differentiation, as with its predecessor for standard QS cocycles, [8, lemma 4.2].

PROPOSITION 5.2. Let $\mathrm{k}$ be an orthogonal direct sum of Hilbert spaces: $\mathrm{k}_{0} \oplus \mathrm{k}_{1}$, let $\varphi \in$ $C B\left(\mathrm{~A} ; B\left(\widehat{\mathrm{k}}_{0}\right)\right)$ and $\psi \in C B(\mathrm{~A} ; B(\widehat{\mathrm{k}}))$ and let $k^{0}=l^{\varphi} \in \mathbb{P}\left(\mathrm{A} ; \mathcal{E}_{\mathrm{k}_{0}}\right)$ and $k=l^{\psi} \in \mathbb{P}\left(\mathrm{A} ; \mathcal{E}_{\mathrm{k}}\right)$ be the respective weak $Q S$ convolution cocycles. Then

$$
k_{t}(a)=k_{t}^{0}(a) \odot P_{\mathrm{k}_{1},[t, \infty[}, a \in \mathrm{A}, \quad t \geqslant 0,
$$

if and only if

$$
\psi(a)=\left[\begin{array}{cc}
\varphi(a) & 0 \\
0 & -\epsilon(a) I_{1}
\end{array}\right], \quad a \in \mathrm{A}
$$

where $I_{1}=I_{\mathrm{k}_{1}}$.

We are ready for the main theorem of this section.

THEOREM 5·3. Let $k \in \mathbb{P}\left(\mathrm{A} ; \mathcal{E}_{\mathrm{k}_{0}}\right)$ be a Markov-regular CPC QS convolution cocycle on a $C^{*}$-bialgebra $\mathrm{A}$. Then there exists another Hilbert space $\mathrm{k}_{1}$, a Markov-regular, *-homomorphic QS convolution cocycle $j \in \mathbb{P}\left(\mathrm{A} ; \mathcal{E}_{\mathrm{k}}\right)$, where $\mathrm{k}:=\mathrm{k}_{0} \oplus \mathrm{k}_{1}$, and a contractive process $W \in \mathbb{P}\left(\mathcal{E}_{\mathrm{k}}\right)$, such that

$$
\widetilde{k}_{t}(a)=W_{t}^{*} j_{t}(a) W_{t}, \quad t \geqslant 0, a \in \mathrm{A},
$$

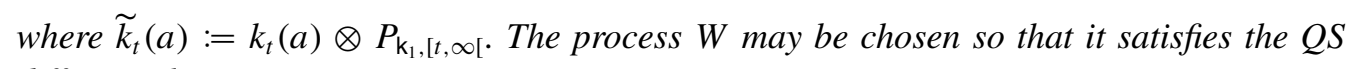
differential equation

$$
\mathrm{d} W_{t}=\left(\tau \otimes U_{t} W_{t}\right) \mathrm{d} \Lambda_{t}, \quad W_{0}=I_{\mathcal{F}_{\mathrm{k}}}
$$

for some $\tau \in B(\hat{\mathrm{k}})$ in which $U \in \mathbb{P}\left(\mathcal{E}_{\mathrm{k}}\right)$ is the projection-valued process given by $U_{t}=j_{t}(1)$, $t \geqslant 0$.

Proof. Let $\varphi \in C B(\mathrm{~A} ; B(\widehat{\mathrm{k}}))$ be the stochastic generator of $k$ (so that $k=l^{\varphi}$ ) and let $(\mathrm{K}, \rho, D, \xi, d, e, t)$ be an associated tuple, as in Theorem 3.3. Set $\mathrm{k}_{1}=\mathrm{K}$ and define $\theta: \mathrm{A} \rightarrow B(\widehat{\mathrm{k}})$ by

$$
\theta(a)=\left[\begin{array}{ccc}
\lambda(a)-t \epsilon(a) & 0 & \delta^{\dagger}(a) \\
0 & -\epsilon(a) I_{0} & 0 \\
\delta(a) & 0 & \rho(a)-\epsilon(a) I_{1}
\end{array}\right], \quad a \in \mathrm{A},
$$

where $I_{i}$ denotes $I_{\mathrm{k}_{i}}, i=0,1$ and $\delta$ is the $(\rho, \epsilon)$-derivation $a \mapsto\left(\rho(a)-\epsilon(a) I_{\mathrm{k}}\right)|\xi\rangle$. The map $\theta$ is completely bounded and as such generates a Markov-regular weak QS convolution cocycle $j=l^{\theta} \in \mathbb{P}\left(\mathrm{A} ; \mathcal{E}_{\mathrm{k}}\right)$. It is easily checked that $\theta$ satisfies the structure relations of Theorem 2.7, so $j$ is *-homomorphic. Now choose any contraction $B \in B\left(\mathrm{k}_{1} ; \mathrm{k}_{0}\right)$ and define

$$
\tau=\left[\begin{array}{ccc}
\frac{1}{2} t & \langle\xi| & 0 \\
0 & -I_{0} & B \\
0 & D & -I_{1}
\end{array}\right] \in B(\widehat{\mathrm{k}})
$$

Then

$$
\tau^{*}+\tau+\tau^{*} \Delta^{Q S} \tau=\left[\begin{array}{ccc}
t & \langle\xi| & 0 \\
|\xi\rangle & D^{*} D-I_{0} & 0 \\
0 & 0 & B^{*} B-I_{1}
\end{array}\right] \leqslant 0,
$$

as $B$ is a contraction, and $\varphi(1) \leqslant 0($ see (3.6)). 
Theorem $5 \cdot 1$ yields the existence of a contractive process $W \in \mathbb{P}\left(\mathcal{E}_{\mathrm{k}}\right)$ satisfying the QS differential equation (5.9) and shows that the process $l \in \mathbb{P}\left(\mathrm{A} ; \mathcal{E}_{\mathrm{k}}\right)$ given by

$$
l_{t}(a)=W_{t}^{*} j_{t}(a) W_{t}, \quad t \geqslant 0, a \in \mathrm{A},
$$

is equal to $l^{\psi}$ where $\psi: \mathrm{A} \rightarrow B(\hat{\mathrm{k}})$ is defined by:

$$
\begin{aligned}
& \psi(a)=\epsilon(a)\left(\tau^{*}+\tau+\tau^{*} \Delta^{Q S} \tau\right)+\left(1+\tau^{*} \Delta^{Q S}\right) \theta(a)\left(1+\Delta^{Q S} \tau\right) \\
& =\epsilon(a)\left[\begin{array}{ccc}
t & \langle\xi| & 0 \\
|\xi\rangle & D^{*} D-I_{0} & 0 \\
0 & 0 & B^{*} B-I_{1}
\end{array}\right] \\
& +\left[\begin{array}{ccc}
1 & 0 & 0 \\
0 & 0 & D^{*} \\
0 & B^{*} & 0
\end{array}\right] \cdot\left[\begin{array}{ccc}
\lambda(a)-t \epsilon(a) & 0 & \delta^{\dagger}(a) \\
0 & -\epsilon(a) I_{0} & 0 \\
\delta(a) & 0 & \rho(a)-\epsilon(a) I_{1}
\end{array}\right] \cdot\left[\begin{array}{lll}
1 & 0 & 0 \\
0 & 0 & B \\
0 & D & 0
\end{array}\right] \\
& =\epsilon(a)\left[\begin{array}{ccc}
t & \langle\xi| & 0 \\
|\xi\rangle & D^{*} D-I_{0} & 0 \\
0 & 0 & B^{*} B-I_{1}
\end{array}\right] \\
& +\left[\begin{array}{ccc}
\lambda(a)-t \epsilon(a) & \delta^{\dagger}(a) D & 0 \\
D^{*} \delta(a) & D^{*} \rho(a) D-\epsilon(a) D^{*} D & 0 \\
0 & 0 & -\epsilon(a) B^{*} B
\end{array}\right] \\
& =\left[\begin{array}{ccc}
\lambda(a) & \delta^{\dagger}(a) D+\epsilon(a)\langle\xi| & 0 \\
D^{*} \delta(a)+\epsilon(a)|\xi\rangle & D^{*} \rho(a) D-\epsilon(a) I_{0} & 0 \\
0 & 0 & -\epsilon(a) I_{1}
\end{array}\right]=\left[\begin{array}{cc}
\varphi(a) & 0 \\
0 & -\epsilon(a) I_{1}
\end{array}\right] .
\end{aligned}
$$

Application of Proposition 5.2 now completes the proof.

Acknowledgements. The author would like to express his gratitude to Martin Lindsay for suggesting the problem considered in this paper and for generous comments improving its final form. The work was partially supported by the Polish KBN Research Grant 2P03A 03024.

\section{REFERENCES}

[1] L. AcCARDi. On the quantum Feynman-Kac formula. Rend. Sem. Mat. Fis. Milano 48 (1978), 135180.

[2] V. P. BelaVkin. Quantum stochastic positive evolutions: characterization, construction, dilation. Comm. Math. Phys. 184 (1997), no. 3, 533-566.

[3] W. R. Bloom and H. HeYer. Harmonic Analysis of Probability Measures on Hypergroups. de Gruyter Studies in Mathematics, 20 (Walter de Gruyter \& Co., 1995).

[4] E. G. EFFros and Z. J. RuAN. Operator Spaces (Oxford University Press, 2000).

[5] Yu. Chapovsky and L. Vainerman. Compact quantum hypergroups. J. Operator Theory 41 (1999), no. 2, 261-289.

[6] U. FRANZ. Lévy processes on quantum groups and dual groups. In Quantum Independent Increment Processes, Vol. II: Structure of Quantum Lévy Processes, Classical Probability and Physics, eds. U. Franz and M. Schürmann. Lecture Notes in Math. 1866 (Springer-Verlag, 2005).

[7] U. FrANZ and M. SchÜRMANN. Lévy processes on quantum hypergroups. In Infinite Dimensional Harmonic Analysis, eds. H. Heyer, T. Hirai and N. Obata (Gräbner, 2000), pp. 93-114.

[8] D. Goswami, J. M. Lindsay and S. J. WiLls. A stochastic Stinespring theorem, Math. Ann. 319 (2001), no. 4, 647-673.

[9] D. Goswami, J. M. Lindsay, K. B. Sinha and S. J. Wills. Dilation of Markovian cocycles on a von Neumann algebra. Pacific J. Math. 211 (2003), 221-247.

[10] A. A. KalyUZHNYI. Conditional expectations on compact quantum groups and new examples of quantum hypergroups. Methods Funct. Anal. Topology 7 (2001), no. 4, 49-68. 
[11] A. A. Kalyuzhny and Yu. A. Chapovsky. A factorization of conditional expectations on Kac algebras and quantum double coset hypergroups. Ukrä̈n. Mat. Zh. 55 (2003), no. 12, 1669-1677; translation in Ukrainian Math. J. 55 (2003), no. 12, 1994-2005.

[12] J. M. LindSAY. Quantum stochastic analysis - an introduction. In Quantum Independent Increment Processes, Vol. I: From Classical Probability to Quantum Stochastics, eds. U. Franz and M. Schürmann. Lecture Notes in Math. 1865 (Springer-Verlag, 2005).

[13] J. M. Lindsay and K. R. PARThaSARATHY. On the generators of quantum stochastic flows. J. Funct. Anal. 158 (1998), 521-549.

[14] J. M. LINDSAY and A. G. SKALSKI. Quantum stochastic convolution cocycles I. Ann. Inst. H. Poincaré Probab. Statist. 41 (2005), no. 3 (En hommage à Paul-André Meyer), 581-604.

[15] J. M. LINDSAY and A. G. SKALSKI. Quantum stochastic convolution cocycles-algebraic and $C^{*}$ algebraic. Banach Center Publ. 73 (2006), 313-324.

[16] J. M. LINDSAY and A. G. SKALSKI. On quantum stochastic differential equations. J. Math. Anal. Appl. 330 (2007), no. 2, 1093-1114.

[17] J. M. LINDSAY and A. G. SKALSKI. Quantum stochastic convolution cocycles II, preprint.

[18] J. M. LinDSAY and S. J. WILLS. Existence, positivity and contractivity for quantum stochastic flows with infinite dimensional noise. Probab. Theory Related Fields 116 (2000), 505-543.

[19] J. M. LINDSAY and S. J. WILLS. Markovian cocycles on operator algebras, adapted to a Fock filtration. J. Funct. Anal. 178 (2000), no. 2, 269-305.

[20] J. M. LindSAY and S. J. WILLS. Existence of Feller cocycles on a $C^{*}$-algebra. Bull. London Math. Soc. 33 (2001), no. 5, 613-621.

[21] J. M. LINDSAY and S. J. WILLS. Homomorphic Feller cocycles on a $C^{*}$-algebra. J. London Math. Soc. (2) 68 (2003), no. 1, 255-272.

[22] M. SchÜrmann. White Noise on Bialgebras. Lecture Notes in Math. 1544 (Springer, 1993). 